\section{PRORATE: A BASIC program for calculating minimum test length given internal consistency constraints}

\author{
MICHAEL J STRUBE \\ Washington University, St. Louis, Missouri
}

When researchers rely on self-report methods, an annoying problem occurs if respondents fail to complete questionnaire items. This problem is particularly troublesome when data are missing on items that are to be combined to produce a composite score (e.g., personality inventories, mood scales, etc.). In such instances, the typical procedure is to estimate the composite score using a prorating method. For example, suppose that a respondent failed to answer 2 items on a 20 -item test. A total score formed by simply adding items would not allow comparison to scores of respondents who completed all 20 items. To achieve comparability, one could calculate the average item response for all respondents or, more typically, calculate the average item response for the subjects with missing data and multiply that average by the number of items in the test. Thus, if each item was scored on a 1- to 5-point scale, and our respondent's average for the 18 items on which responses were made was 3.5 , then the prorated or estimated full length total would be 20 * 3.5 or 70 .

Address correspondence to Michael J Strube, Department of Psychology, Washington University, St. Louis, MO 63130.
One major problem is deciding how much missing data to allow. As missing data increases, the reliability of a respondent's total score decreases. Although one could resort to a percentage rule (e.g., allow no more than $20 \%$ missing items), a more defensible alternative is available. Specifically, given the internal consistency of the full test (which can be calculated from the available data), a modification of the Spearman-Brown prophesy formula (see Nunnally, 1978) can be used to estimate the internal consistency of a truncated or prorated test. Alternately, one can specify in advance the minimum allowable internal consistency and calculate the minimum scale length that one can use. If a respondent had fewer complete items than this minimum, then that respondent's data would not be used in subsequent statistical analyses.

This program allows the user two options: (1) to specify a specific minimum internal consistency in response to which the program calculates the minimum allowable test length, or (2) to list the estimated internal consistency for tests of length 1 through $k$, where $k$ is the number of items on the full-length test. Input for the program is the fulllength internal consistency and test length. In the case of Option 1, the user must also specify a minimum internal consistency.

The program is IBM-PC compatible. A listing of the program is available free of charge from the author.

\section{REFERENCE}

Nunnally, J. C. (1978). Psychometric theory (2nd ed.). New York: McGraw-Hill. 